\title{
Spatio-temporal Rasch analysis of quality of life outcomes in the French general population. Measurement invariance and group comparisons
}

\author{
Jean-Benoit Hardouin ${ }^{1,2^{*}}$, Etienne Audureau ${ }^{3,4}$, Alain Leplège ${ }^{3,5}$ and Joël Coste 3,4
}

\begin{abstract}
Background: This study aims at analyzing Health related quality of life (HRQOL) data on the French general population between 1995 and 2003 using an Item Response Theory (IRT) model.

Methods: Data concerned 26388 individuals having responded to the SF36 questionnaire in 1995 or in 2003. General Health, Mental Health and Physical Functioning dimensions have been analyzed using a latent regression mixed Partial Credit Model. Differential Item Functioning (DIF) have been searched on each item between age categories, genders, regions of residency, and years of study. Mean and variance of the latent traits have been explained by the same variables, in order to quantify their impact.

Results: Few DIF have been detected between age categories or genders. The analysis shows already known evolutions for HRQoL data: the decrease with age and the differences between genders with worst values for women. We note differences between regions, with better mean value in Paris, in the West or in the South of France, and worst values in the North and in the East. Last, a decrease of the three studied dimensions is noted between 1995 and 2003.

Conclusions: This study using IRT model offers several advantages compared to a classical approach based on scores. First, DIF can be taken into account. More, handling of missing data is easy, because IRT models do not required imputation of missing data. Last, analysis using IRT model is more powerful than analysis based on scores, and allow highlighting a most important number of effects.
\end{abstract}

\section{Background}

There is considerable interest in measuring and monitoring Health-Related Quality of Life (HRQoL) in general populations. This yields results complementary to traditional mortality and morbidity indicators that could be useful when assessing health disparities or measuring the efficacy of healthcare policies [1-3]. However, monitoring HRQoL through time and space (regions or states within a country, countries) requires the use of measurement instruments which have the crucial property of invariance: the instrument should give the same score for

\footnotetext{
*Correspondence: jean-benoit.hardouin@univ-nantes.fr

${ }^{\dagger}$ Equal contributors

'UPRES EA 4275 (SPHERE) « Biostatistics, Pharmacoepidemiology and Subjective Measures in Health Sciences », University of Nantes, Nantes, France

${ }^{2}$ Plateforme de Biométrie, Direction de la Recherche Clinique, CHU de Nantes, Nantes, France

Full list of author information is available at the end of the article
}

a subject with a given level of HRQoL whatever the time and the place of the measurement. This property is a necessary to assert that differences are genuine and not related to artifacts or other problems in the measurement process.

Item Response Theory (IRT) models, and in particular the models of the Rasch family, are increasingly employed for the validation or the reduction of measurement instruments, and in particular those for HRQoL. These models have, however, been rarely used for analysis of HRQoL datasets [4], even though many measurement instruments have now been validated using this modern measurement theory. Rasch analyses provide unique opportunities to assess simultaneously the invariance of measures and differences between groups. Such analyses also allow the detection and handling of differential item functioning (DIF) [5]: items function differently if they yield different responses patterns across 
groups of subjects. In addition, the specific objectivity property of the Rasch family models allow obtaining an estimation of the latent concept independent of the answered items of each individual and allows simple handling of missing data, without any imputation being required. These models can be used to perform a latent regression, allowing the explanation of the latent variable by external covariates [6,7]. This latent regression is easy to interpret because, in IRT models, the measured latent trait acquires interval measurement properties [6], i.e. a difference in this latent trait can be interpreted in the same way whatever its level on this latent trait. Last, the homoscedasticity assumption can be relaxed by introducing covariates explaining the value of the variance of the latent trait. This assumption is required when analyzing data with linear models and supposes that the dispersion (variance) of analyzed variable is constant whatever the level of the covariates in the model.

In term of public Health, there is increasing evidence of possible worsening trends in HRQoL in some western countries $[1,8,9]$. In France, work using Classical Test Theory (CTT) reveals differences through time (19952003) and space (administrative regions) [3]. We assessed the independence of these findings from invariance-related issues that could occur in the measurement process. We therefore tested the hypotheses that quality of life differs between regions and has changed between 1995 and 2003 in France; we used a latent regression Rasch model approach, after searching and, if necessary, accounting for DIF for particular covariates (age, gender, region and year of survey) to obtain invariance of measurement for the comparisons between groups of interest in the latent regression model. Here, we present the step-by-step methodology employed and the results for three distinctive dimensions of the MOS-SF36 questionnaire, using data from two large representative surveys of the French population.

\section{Methods}

\section{Samples}

We used two large population-based surveys conducted in France, both representative of the French population [3]. The first was conducted by the SOFRES polling agency in 1995 and included 3656 subjects. The second was conducted by INSEE (French Institute of Statistics and Economic Studies) in 2003 and included 23,018 subjects having completed at least one item of the SF-36 questionnaire. Only subjects aged between 18 and 84 years were retained in the study, giving a total of 26,388 subjects. For each subject, gender, age, and region of residency were recorded (see Figure 1). The age variable was categorized as follows: 18-24, 25-34, 35-44, 45-54, 55-64, 65-74, 75-84 years.

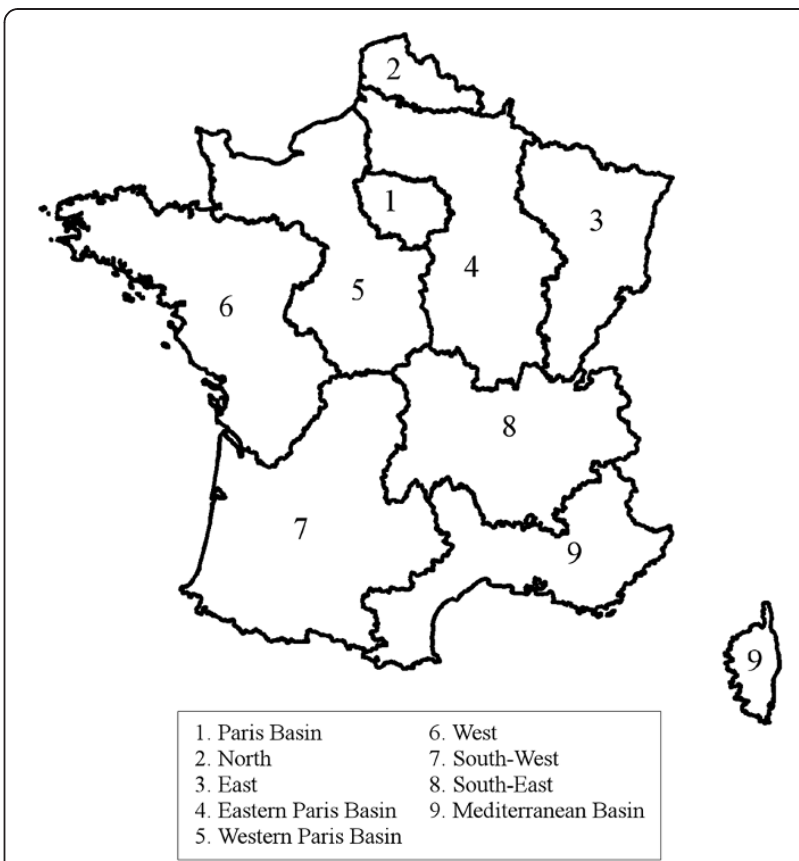

Figure 1 Map of the nine French regions considered in this analysis.

\section{Questionnaires}

For each subject, the responses to the French version of the SF-36 questionnaire $[10,11]$ were available. This questionnaire was developed and validated as part of the International Quality of Life Assessment (IQOLA) project [12]. It is made up of 35 questions divided into eight scales: physical functioning (10 items), role limitations relating to physical health (4 items), bodily pain (2 items), general health perceptions (5 items), vitality (4 items), social functioning (2 items), role limitation relating to mental health (3 items), and mental health (5 items). One additional item assesses changes in health in the last year. The answer to each question was rated on an ordinal scale of between 2 and 6 points. This questionnaire is short and quick to administer (5-10 min) and well-adapted for studies in general populations. It has already been validated with models of the Rasch family [13,14].

\section{Statistical analyses}

The statistical analysis was conducted in three stages as follows: 1. DIF on items was investigated between genders, age categories, region and year of survey; 2 . Assumptions of IRT were studied using non parametric Item Response Theory; 3. Partial Credit Models were fitted to data in each dimension of the SF36, and covariates explaining the mean and the variances of the latent trait were tested. 


\section{Differential Item Functioning detection}

A first analysis has been performed to detect DIF for the variables gender, age, region and year of survey. For each combination of gender ( 2 categories), age (7 categories) and year of survey (2 categories) (28 strata), a Partial Credit Model (PCM) [15] was fitted. This model can be written

$$
P\left(Y_{n j}=y / \theta_{n}, \boldsymbol{\delta}_{\mathbf{j}}\right)=\frac{\exp \left(y \theta_{n}-\sum_{l=1}^{y} \delta_{j l}\right)}{\sum_{c=0}^{m_{j}} \exp \left(c \theta_{n}-\sum_{l=1}^{c} \delta_{j l}\right)}
$$

Where $Y_{n j}$ is the response variable of the nth individual $(n=1 \ldots N)$ to the $j$ th item $(j=1 \ldots J), \delta_{j 1}$ is the difficulty parameter associated to the lth response category $\left(\mathrm{l}=1 \ldots \mathrm{m}_{\mathrm{j}}\right)$ of the $\mathrm{jth}$ item $(\mathrm{j}=1 \ldots \mathrm{J})$, and $\theta_{\mathrm{n}}$ $(\mathrm{n}=1 \ldots \mathrm{N})$ is a set of parameters representing the values of the latent trait for the individuals; $\delta_{j 1}$ were estimated by the Pairwise Conditional Estimation (PCE) method [16].

In each of the 28 strata, the location associated to each item $\left(\frac{1}{m_{j}} \sum_{l=1}^{m_{j}} \hat{\delta}_{j l}\right)$ was estimated. An ANOVA weighted by the sample size used for each model was fitted for each item location by using the gender, the age and the sample as factors, and no interaction. Gender and age effects were studied first. A DIF was considered large if it was significant at $5 \%$ and caused a difference greater than 0.1 in the estimation of the mean location between the different levels of a factor.

In a second step, the DIF effects of the region (9 categories) and of the year of survey (2 categories) were evaluated, by estimating the locations of each item for each region and each year (18 models), and by taking into account gender and age effects if DIF had been detected in the previous step.

\section{Non parametric Item Response Theory: verification of assumptions}

After this DIF detection step, we subjected the data to non parametric Item Response Theory analysis [17]. First, a Monotonely Homogeneous Model of Mokken (MHMM) was fitted to the data. If this model has a good fit, the three fundamental assumptions of the Item Response Theory (IRT) (unidimensionality, local independence and monotonicity) are considered to be verified. This analysis also indicates possibly problematic items and or/subjects. The fit of the model was evaluated using Loevinger's $\mathrm{H}$ coefficients. Three kinds of $\mathrm{H}$ coefficients are used to validate the fit of MHMM: $\mathrm{H}$ (the coefficient of scalability), $\mathrm{H}_{\mathrm{j}}$ (the coefficients associated to each item $j ; j=1, \ldots, J$ ) and $H_{j k}$ (the coefficients associated to each pair of items $j, k, j=1 \ldots J, k=1 \ldots J)$. A MMHM is considered to have a correct fit if $\mathrm{H}>0.3, \mathrm{Hj}>0.3$ and $\mathrm{Hjk}>0$ [17]. The respect of the monotonicity hypothesis was assessed with a specific criterion $\left(\mathrm{Crit}_{\mathrm{M}}\right)$; monotonicity is respected if $\mathrm{Crit}_{\mathrm{M}}$ is below 40 for each item [18].

\section{Analysis of the latent trait}

For each item, we created a pseudo-item for each level of the variables gender, age category, region and study year which presented a DIF effect [19]. This pseudoitem was given a value equal to the responses of the subjects with this level on the factor and was replaced by a missing value for the other subjects.

A PCM was then fitted on the set of the pseudoitems. In this new model, a random variable is used to estimate the latent trait. This random variable is assumed to be distributed as a normal distribution with mean $\mu$ and variance $\sigma^{2}$. Since an identifiability constraint is required, $\mu$ is set to 0 . At this step, the PCM was fitted to the data. The sample was very large, and fit tests based on a chi-squared comparison between observed and expected frequencies are highly susceptible to large sample sizes [20]. Therefore, the sample size was artificially set at 500 individuals to evaluate the fit for a sample of moderate size by adjusting proportionally the statistics of the test (that is to say, by multiplying it by $500 / \mathrm{N}$ ) [21]. This procedure allows avoiding detection of a significant but small and irrelevant gap between the model and the data, as possible with large sample sizes. Indeed, a sample size of only several hundreds of individuals is usually considered as acceptable in order to test the fit of a PCM $[20,22,23]$.

The estimations of the item parameters were set to the estimated values obtained in this model, allowing analysis of the effects of the variables gender, age, region and year of survey on the mean of the latent trait. These variables and their interactions of order 2 were consequently introduced into a latent regression PCM to explain the mean of the latent variable [6]. These variables and interactions were removed one at a time from the model if they were not significant at $5 \%$ and if their removal did not increase the value of Akaike's criterion (AIC) [24]

The variables gender, age, region and year of survey (and their interaction of order 2) were further introduced into the model to explain the variance of the latent trait, and consequently, to account for the heteroscedasticity between groups of subjects. These variables were removed one at a time if they were not significant at 5\% and if their omission did not increase the value of Akaike's criterion (AIC) [24]. 
The model obtained can be written, as proposed by Boeck and Wilson [6], thus:

$$
\begin{aligned}
& P\left(Y_{n j}=y / \Theta_{n}\right)=\frac{\exp \left(y \theta_{n}-\sum_{l=1}^{y} \hat{\delta}_{j l}\right)}{\sum_{c=0}^{m_{j}} \exp \left(c \theta_{n}-\sum_{l=1}^{c} \hat{\delta}_{j l}\right)} \\
& \Theta_{n} \sim N\left(\mu_{n}, \sigma_{n}^{2}\right) \\
& \mu_{n}=\mu^{*}+X_{n} \beta_{\mu}+\varepsilon_{\mu, n} \\
& \sigma_{n}^{2}=\sigma^{2 *}+Z_{n} \gamma_{\sigma^{2}}+\varepsilon_{\sigma^{2}, n}
\end{aligned}
$$

Where $Y_{n j}$ is the response variable of the nth individual $(n=1 \ldots N)$ to the $j$ th item or pseudo-item $(j=1 \ldots J)$, $\Theta_{n}$ is the latent variable representing the latent trait for the $n$th individual $(\mathrm{n}=1 \ldots \mathrm{N}), \hat{\delta}_{j l}$ is the estimated difficulty parameter associated with the lth $\left(l=1 \ldots \mathrm{m}_{\mathrm{j}}\right)$ response category of the $j$ th item or pseudo-item $(j=1 \ldots J)$, $X_{n}$ is a set of covariates explaining the mean of the latent variable $\mu$, and $\beta_{\mu}$ is the set of parameters associated with these covariates, $Z_{n}$ is a set of covariates explaining the variance of the latent variable $\sigma^{2}$, and $\gamma \sigma^{2}$ is the set of parameters associated with these covariates. $\mu^{*}$ and $\sigma^{2 *}$ are the estimations of the mean and of the variance in a reference group (men, 18-24 years, Paris basin, year 1995). The $\hat{\delta}_{j l}$ parameters are estimated under the constraint $\mu=0$, such that the global mean of the latent trait is 0 .

To evaluate the relative importance of the covariates in explaining the latent trait, we computed the explained variance as follows: let $\hat{\sigma}_{0} 2$ be the estimated value of the variance of the latent trait in the model without covariates (a model with po parameters) and $\hat{\sigma}^{2}$ the estimated value of the variance of the latent trait in the model with the selected covariates that explain the mean of the latent trait (included in the $X_{n}$ matrix - a model with $k$ parameters); the rate $\tau$ of explained variance can be computed as:

$$
\tau=\frac{1-(n-1-k) \hat{\sigma}^{2}}{\left(n-1-k_{0}\right) \hat{\sigma}_{0}^{2}}
$$

\section{Studied dimensions}

The three most used dimensions of the SF-36 were studied: General Health (GH), Physical Functioning (PF) and Mental Health (MH) (See Additional file 1 for details).

The General Health dimension is composed of five items with five response categories. Three items of this dimension must be inversed $[1,3,5]$. To analyse the data by IRT, the responses of the item 1 were not weighted and consequently, for each item, the responses were simply coded from 0 to 4 .

The Physical Functioning dimension is composed of 10 items with three response categories. None of them need to be inversed and all the response categories were coded from 0 to 2 . Three of the sets of items are not locally independent, so recoding of these items was necessary [25] (See Additional file 2 for details).

The Mental Health dimension is composed of five items with six response categories. None of them need to be inversed and all the response categories were coded from 0 to 5 .

\section{Software}

The software used included Stata 11 MP [26] (Non parametric IRT analysis [27] and DIF detection), RUMM 2030 (fit of the model) [21] and SAS 9.2 [28] (analysis of the latent trait with the macro-program \%Anaqol [29] and the NLMIXED procedure).

\section{Results}

\section{Descriptive analysis}

The common dataset, consisting of data from the two surveys, corresponded to 12272 (46.5\%) men and 14116 $(53.5 \%)$ women. The average age was 46.7 years (SD: 17.0). The distribution of the subjects into regions was: $21 \%$ in the Paris basin, $10 \%$ in the North, $8 \%$ in the East, $15 \%$ in the Eastern Paris Basin, 7\% in the Western Paris Basin, 11\% in the West for, South-8\% in the West for, 9\% in the South-East for and $11 \%$ in the Mediterranean Basin.

\section{Analysis of the Differential Item Functionning}

Table 1 presents the results of the DIF tests for each item of the three dimensions studied, and, for significant DIF, the maximal differences between item locations.

The three dimensions studied include 16 items, six of which presented DIF. The first two items of the GH dimension presented DIF between age categories (18-44, 45-64 and 65-84 for GH1; 18-34, 35-64, 65-84 for GH2). The difficulties parameters increased with age for GH1 but decreased with age for GH2.

For the PF dimension, PF3 presented DIF between genders (women considered this item as more difficult than men); PF3 and PF45 presented DIF between year of study (difficulty parameters in 2003 were lesser than these ones in 1995); and PF6 presented DIF between age categories (18-34 and 35-84: difficulty parameters increased with age).

Last, for the MH dimension, only MH1 presented DIF between age categories $(18-44,45-54,55-64,65-74$ and 75-84: difficulty parameters decreased with age).

For the following analyses, the items presenting DIF for a variable have been replaced by two or more pseudo-items taking the values of the initial items for the corresponding level of this variable, and missing values for the other levels of this variable. Consequently, 
Table 1 Maximal difference of the averaged item locations and associated p-value for each item of the three dimensions studied, and for the variables gender, age, region and year of survey

\begin{tabular}{|c|c|c|c|c|c|c|c|c|c|}
\hline & & \multicolumn{2}{|c|}{ Gender } & \multicolumn{2}{|l|}{ Age } & \multicolumn{2}{|c|}{ Region } & \multicolumn{2}{|c|}{ Year of survey } \\
\hline & & Diff $^{*}$ & $p^{\$}$ & $\overline{\text { Diff* }^{*}}$ & $p^{5}$ & $\overline{\text { Diff }^{*}}$ & $p^{5}$ & Diff* & $p^{\$}$ \\
\hline \multirow[t]{5}{*}{ General Health } & $\mathrm{GH} 1$ & - & 0.77 & 0.70 & 0.0055 & - & 0.98 & - & 0.23 \\
\hline & GH11a & - & 0.20 & 1.01 & 0.0049 & - & 0.95 & - & 0.14 \\
\hline & $\mathrm{GH} 11 \mathrm{~b}$ & - & 0.80 & - & 0.084 & - & 0.77 & - & 0.38 \\
\hline & GH11c & - & 0.065 & - & 0.39 & - & 0.14 & - & 0.38 \\
\hline & $\mathrm{GH} 11 \mathrm{~d}$ & - & 0.18 & - & 0.71 & - & 0.19 & - & 0.50 \\
\hline \multirow[t]{6}{*}{ Physical Functioning } & PF12 & - & 0.84 & - & 0.97 & - & 0.90 & - & 0.10 \\
\hline & PF3 & 0.60 & 0.005 & - & 0.26 & - & 0.51 & 0.16 & 0.0264 \\
\hline & PF45 & - & 0.84 & - & 0.87 & - & 0.79 & 0.17 & 0.0418 \\
\hline & PF6 & - & 0.97 & 0.41 & 0.0498 & - & 0.90 & - & 0.070 \\
\hline & PF789 & - & 0.56 & - & 0.067 & - & 0.81 & - & 0.14 \\
\hline & PF10 & - & 0.23 & - & 0.99 & - & 0.99 & - & 0.23 \\
\hline \multirow[t]{5}{*}{ Mental Health } & $\mathrm{MH} 1$ & - & 0.89 & 0.77 & 0.0003 & - & 0.86 & - & 0.89 \\
\hline & $\mathrm{MH} 2$ & - & 0.68 & - & 0.93 & - & 0.99 & - & 0.13 \\
\hline & $\mathrm{MH3}$ & - & 0.88 & - & 0.56 & - & 0.51 & - & 0.22 \\
\hline & $\mathrm{MH} 4$ & - & 0.35 & - & 0.71 & - & 0.99 & - & 0.95 \\
\hline & MH5 & - & 0.21 & - & 0.18 & - & 0.48 & - & 0.19 \\
\hline
\end{tabular}

* : maximal difference in absolute value between the estimated locations for each item between two levels of the variable studied.

$\$: p$-value associated to the maximal difference between the estimated locations for each item between two levels of the variable studied.

9, 10 and 9 items or pseudo-items were analysed for the dimensions $\mathrm{GH}, \mathrm{PF}$ and $\mathrm{MH}$, respectively.

\section{PCM without covariates explaining the latent traits}

Non-parametric IRT analysis did not detect any violation of the fundamental assumptions of the IRT for any of the dimensions studied. The fit tests were not significant with a moderate sample size $(\mathrm{N}=500)$ for all dimensions (Table 2).

Graphical assessment indicated that the latent traits for the three dimensions followed a normal distribution (not shown). For each dimension, the range of values of the difficulty parameters were similar to the range of the latent trait. This demonstrated that the items were adapted to the samples.
PCM with covariates explaining the latent trait -

\section{Construction of the final models}

The first step in the construction of the models explaining the latent trait was to recode the variable age such that it could be handled as a quantitative (rather than ordinal) variable. The values were chosen to be coherent with the values of the parameters associated with the dummy variables representing each age category in the preceding analysis.

Tables 3, 4 and 5 present the models obtained for the "General Health", "Physical Functioning" and "Mental Health" dimensions, respectively. For each model, the constants (for the mean and the variance) represent the mean and the variance of the reference group (men, 18-24 years, Paris Basin, year 1995).

Table 2 Fit tests for the modelling of each dimension by a Partial Credit Model (PCM) with the complete sample or with a sample fixed to 500

\begin{tabular}{|c|c|c|c|c|c|c|c|c|c|c|}
\hline & \multicolumn{4}{|c|}{ Non parametric IRT analysis } & \multicolumn{3}{|c|}{$\begin{array}{l}\text { Fit of a PCM for the } \\
\text { complete sample }(n=26388)\end{array}$} & \multicolumn{3}{|c|}{$\begin{array}{l}\text { Fit of a PCM for a fixed, } \\
\text { moderate sample size }(n=500)\end{array}$} \\
\hline & $\mathrm{H}$ & $\operatorname{Min}\left(\mathrm{H}_{\mathrm{j}}\right)$ & $\operatorname{Min}\left(\mathrm{H}_{\mathrm{jk}}\right)$ & $\operatorname{Max}\left(\mathrm{Crit}_{M}\right)$ & $x^{2}$ & df & $\mathbf{P}$ & $x^{2}$ & df & $p$ \\
\hline General Health & $0.49^{*}$ & $0.40^{*}$ & $0.31^{*}$ & -5 & 1320.42 & 81 & $<0.0001$ & 39.36 & 81 & 0.99 \\
\hline Physical Functioning & $0.76^{*}$ & $0.72^{*}$ & $0.66^{*}$ & -21 & 3895.46 & 94 & $<0.0001$ & 103.64 & 94 & 0.23 \\
\hline Mental Health & $0.57^{*}$ & $0.50^{*}$ & $0.33^{*}$ & -10 & 2808.10 & 81 & $<0.0001$ & 55.95 & 81 & 0.98 \\
\hline
\end{tabular}

*: significant at $5 \%$. 
Table 3 Estimation of the parameters associated with the covariates explaining the mean of the latent trait "General Health" $\left(\beta_{\mu}\right)$ and with the covariates explaining the variance of the latent trait $\left(\gamma \sigma^{2}\right)$

\begin{tabular}{|c|c|c|c|c|}
\hline & Effect & Estimate & Standard error & p-value \\
\hline \multirow{13}{*}{$\begin{array}{l}\text { Variables explaining the } \\
\text { mean of the latent trait }\left(\beta_{\mu}\right)\end{array}$} & Constant $\left(\mu^{*}\right)$ & 0.8238 & 0.0415 & $<0.0001$ \\
\hline & Women & -0.2121 & 0.0267 & $<0.0001$ \\
\hline & Survey 2003 & -0.1393 & 0.0413 & 0.0007 \\
\hline & Ager & -0.0349 & 0.0019 & $<0.0001$ \\
\hline & East & -0.2039 & 0.0480 & $<0.0001$ \\
\hline & West & 0.1259 & 0.0344 & 0.0003 \\
\hline & South-West & -0.0666 & 0.0301 & 0.0272 \\
\hline & Ager*Women & 0.0049 & 0.0013 & 0.0002 \\
\hline & Ager*sample2003 & -0.0030 & 0.0018 & 0.1047 \\
\hline & Ager* (North, West) & -0.0046 & 0.0013 & 0.0004 \\
\hline & Ager* (East) & 0.0062 & 0.0024 & 0.0096 \\
\hline & Survey 2003*(North, Eastern Paris Basin) & -0.1172 & 0.0233 & $<0.0001$ \\
\hline & Survey 2003*(Western Paris Basin, South-East) & -0.0675 & 0.0266 & 0.0113 \\
\hline \multirow{6}{*}{$\begin{array}{l}\text { Variables explaining the } \\
\text { variance of the latent trait }\left(\gamma \sigma^{2}\right)\end{array}$} & Constant $\left(\sigma^{2 *}\right)$ & 1.4130 & 0.0791 & $<0.0001$ \\
\hline & Survey 2003 & -0.1499 & 0.0837 & 0.0730 \\
\hline & Ager & -0.0068 & 0.0034 & 0.0439 \\
\hline & East & -0.2334 & 0.0513 & $<0.0001$ \\
\hline & South-West & -0.1254 & 0.0534 & 0.0188 \\
\hline & Ager*Survey 2003 & 0.0068 & 0.0037 & 0.0632 \\
\hline \multirow[t]{3}{*}{ Explained variance } & Estimated variance without covariates: $\hat{\sigma}_{0} 2\left(\mathrm{k}_{0}\right)$ & $1.4272(37)$ & & \\
\hline & Estimated variance with covariates: $\hat{\sigma}^{2}(\mathrm{k})$ & $1.2406(50)$ & & \\
\hline & Rate of explained variances: $\tau$ & $13 \%$ & & \\
\hline
\end{tabular}

Ager is a categorical variable taking values 0 for the $18-24,2$ for the $25-34,12$ for the $35-44,17$ for the $45-54$, 24 for the $55-64$, 32 for the $65-74$, and 40 for the 75-84 year-olds.

Women is a variable coded 1 for women and 0 for men.

Survey 2003 is coded 1 for the year 2003 and 0 for year 1995.

Region names are dummy variables coded 1 for the cited region and 0 for other geographical areas.

\section{General Health}

Women gave a lower score than men on the latent trait (for example, -0.21 for the younger age groups). The value of the latent trait decreased with age for men: In 1995 , relative to the 18-24 year-old group, the value was -0.07 for the $25-34,-0.42$ for the $35-44,-0.59$ for the $45-54,-0.84$ for the $55-64,-1.12$ for the $65-74$ and -1.40 for the $75-84$ year-old groups. There was a similar trend for women, although the decline in value was lower: In 1995, relative to the 18-24 year-old group, the value was -0.06 for the $25-34,-0.36$ for the $35-44,-0.41$ for the $45-54,-0.72$ for the $55-64,-0.96$ for the $65-74$ and -1.20 for the $75-84$ year-old groups. These trends were stronger in 2003: 75-84 year old men had a value 1.52 lower, and 75-84 year old women a value 1.32 lower than the respective 18-24 year-olds. These trends were significantly stronger in the North and West regions and weaker in the East region. The trends of the mean of the latent trait as a function of the age categories, according to gender and year of survey are given in Figure 2a.

The latent traits in 1995 in the South West region and East region were lower $(-0.07$ and -0.20 , respectively, than in the Paris Basin. Region 6 (West) presented a greater level on the latent trait in $1995(+0.13)$ compared to Paris. The decrease between 1995 and 2003 of the mean latent trait was larger in the North region and Eastern Paris Basin, and to a lesser extent, in the Western Paris Basin and South-East region than in the Paris region.

The variance of the latent trait in the reference group (men, 18-24 year-olds, Paris, 1995)was estimated to be 1.4130 . The estimated variance of the latent trait for the 18-24 year-old group in 2003 was lower (average, -0.15). The variance decreased with age in 1995 (0.27 lower for the 75-84 than 18-24 year-olds), but not in 2003 (the age effect $(-0.0068)$ was equal to the parameter associated with the interaction age*sample (0.68)). The 
Table 4 Estimation of the parameters associated with the covariates explaining the mean of the latent trait "Physical Functioning" $\left(\beta_{\mu}\right)$ and with the covariates explaining the variance of the latent trait $\left(\gamma \sigma^{2}\right)$

\begin{tabular}{|c|c|c|c|c|}
\hline & Effect & Estimate & Standard Error & p-value \\
\hline \multirow{9}{*}{$\begin{array}{l}\text { Variables explaining the } \\
\text { mean of the latent trait }\left(\beta_{\mu}\right)\end{array}$} & Constant $\left(\mu^{*}\right)$ & 3.1131 & 0.0834 & $<0.0001$ \\
\hline & Women & -1.1154 & 0.0816 & $<0.0001$ \\
\hline & Survey 2003 & -0.2447 & 0.0512 & $<0.0001$ \\
\hline & Ager & -0.5960 & 0.0110 & $<0.0001$ \\
\hline & North & -0.2809 & 0.0696 & $<0.0001$ \\
\hline & Western Paris Basin & -0.1386 & 0.0723 & 0.0554 \\
\hline & West, South-West, South-East & 0.3284 & 0.0525 & $<0.0001$ \\
\hline & Women*Ager & 0.0667 & 0.0127 & $<0.0001$ \\
\hline & $\begin{array}{l}\text { Ager*(North, Eastern Paris Basin, West, } \\
\text { South-West, South-East) }\end{array}$ & -0.0345 & 0.0079 & $<0.0001$ \\
\hline \multirow{6}{*}{$\begin{array}{l}\text { Variables explaining the } \\
\text { variance of the latent trait }\left(\gamma \sigma^{2}\right)\end{array}$} & Constant $\left(\sigma^{2 *}\right)$ & 8.9934 & 0.5003 & $<0.0001$ \\
\hline & Women & -2.4176 & 0.4027 & $<0.0001$ \\
\hline & Survey 2003 & 3.0001 & 0.4436 & $<0.0001$ \\
\hline & Ager & -0.5758 & 0.0650 & $<0.0001$ \\
\hline & Women*Ager & 0.1404 & 0.0533 & 0.0084 \\
\hline & Survey $2003^{*}$ Ager & -0.2560 & 0.0595 & $<0.0001$ \\
\hline \multirow[t]{3}{*}{ Explained variance } & Estimated variance without covariates: $\hat{\sigma}_{0} 2\left(k_{0}\right)$ & $9.3182(21)$ & & \\
\hline & Estimated variance with covariates: $\hat{\sigma}^{2}(\mathrm{k})$ & $6.8119(30)$ & & \\
\hline & Rate of explained variances: $\tau$ & $27 \%$ & & \\
\hline
\end{tabular}

Ager is a categorical variable taking values 0 for the $18-24,1$ for the $25-34,2$ for the $35-44,4$ for the $45-54,6$ for the $55-64,8$ for the $65-74$, and 10 for the 75-84 year-old groups.

Women is a variable coded 1 for women and 0 for men.

Survey 2003 is coded 1 for the year 2003 and 0 for year 1995.

Region names are dummy variables coded 1 for the cited region and 0 for other geographical areas.

variance was lower in the East and South West regions than in the Paris region.

\section{Physical Functioning (PF)}

Women presented a lower latent trait estimation than men $(-1.12$ for the $18-24)$. The level of the latent trait decreased with age for men (relative to the 18-24 yearold group: -0.60 for the $25-34,-1.19$ for the $35-44,-2.38$ for the $45-54,-3.58$ for the $55-64,-4.77$ for the $65-74$ and -5.96 for the $75-84$ year-old groups), and to a lesser extent for women (relative to the 18-24 year-old group: -0.53 for the $25-34,-1.06$ for the $35-44,-2.12$ for the $45-54,-3.18$ for the $55-64,-4.23$ for the $65-74$ and -5.29 for the $75-84$ year-old groups). The latent trait mean decreased between 1995 and 2003 (-0.24).

The latent trait estimation in the West, South West and South-East regions were higher $(+0.33)$, and in North $(-0.28)$ and Western Paris Basin $(-0.14)$ lower than that in Paris. The age trend was more pronounced in the North, Eastern Paris Basin, West, South West and South East regions than in Paris region for men, relative to the 18-24 year-old group, -0.63 for the $25-34,-1.26$ for the $35-44,-2.52$ for the $45-54,-3.78$ for the $55-64$,
-5.04 for the $65-74$ and -6.31 for the $75-84$ year-old groups.

The estimated values of the mean of the latent trait "Physical Functioning" as a function of age class, for each gender, in 1995 and 2003, in Paris Basin are given in Figure 2b.

The variance in the reference group (men, 18-24, Paris, 1995) was estimated to be 8.99. The variance was smaller for women (-2.42), and larger in $2003(+3.00)$. The variance decreased with age $(-0.58$ for an increase of 1 in the recoded variable age), and this trend is weaker for women in $1995(-0.43)$ and larger in 2003 ( -0.73 for men and -0.59 for women).

\section{Mental Health (MH)}

Women presented a lower latent trait estimation than men (-0.47). This difference is less pronounced in the Centre West than other regions $(-0.32)$. The latent trait estimation decreased with age (relative to the 18-34 year-old group: -0.09 for the $35-44,-0.19$ for the $45-64$, and -0.28 for the $64-74,-0.56$ for the 75-84 year-old groups). The latent trait value decreased between 1995 and 2003 (-0.18). 
Table 5 Estimation of the parameters associated with the covariates explaining the mean of the latent trait "Mental Health" $\left(\beta_{\mu}\right)$ and with the covariates explaining the variance of the latent trait $\left(\gamma \sigma^{2}\right)$

\begin{tabular}{|c|c|c|c|c|}
\hline & Effect & Estimate & Standard error & $p$-value \\
\hline \multirow{9}{*}{$\begin{array}{l}\text { Variables explaining the } \\
\text { mean of the latent trait }\left(\beta_{\mu}\right)\end{array}$} & Constant $\left(\mu^{*}\right)$ & 0.5924 & 0.0313 & $<0.0001$ \\
\hline & Women & -0.4654 & 0.0186 & $<0.0001$ \\
\hline & Survey 2003 & -0.1824 & 0.0264 & $<0.0001$ \\
\hline & Ager & -0.0936 & 0.0076 & $<0.0001$ \\
\hline & North, Western Paris Basin & -0.1650 & 0.0319 & $<0.0001$ \\
\hline & East, Eastern Paris Basin & -0.0872 & 0.0238 & 0.0002 \\
\hline & West, South-West & 0.1110 & 0.0319 & 0.0005 \\
\hline & Ager*(North, West, South-West) & -0.0425 & 0.0132 & 0.0005 \\
\hline & Women*(Western Paris Basin) & 0.1421 & 0.0552 & 0.0101 \\
\hline \multirow{5}{*}{$\begin{array}{l}\text { Variables explaining the } \\
\text { variance of the latent trait }\left(\gamma \sigma^{2}\right)\end{array}$} & Constant $\left(\sigma^{2 *}\right)$ & 1.6797 & 0.0471 & $<0.0001$ \\
\hline & Ager & 0.1080 & 0.0147 & $<0.0001$ \\
\hline & North, Eastern and Western Paris Basins, South-East & -0.1349 & 0.0530 & 0.0110 \\
\hline & East, West, South-West & -0.2305 & 0.0562 & $<0.0001$ \\
\hline & Mediterranean Basin & 0.1549 & 0.0792 & 0.0505 \\
\hline \multirow[t]{3}{*}{ Explained variance } & Estimated variance without covariates: $\hat{\sigma}_{0} 2\left(k_{0}\right)$ & $1.8377(46)$ & & \\
\hline & Estimated variance with covariates: $\hat{\sigma}^{2}(\mathrm{k})$ & $1.7436(54)$ & & \\
\hline & Rate of explained variances: ${ }_{\tau}$ & $5 \%$ & & \\
\hline
\end{tabular}

Ager is a categorical variable taking values 0 for the $18-34,1$ for the $35-44,2$ for the $45-64,3$ for the $65-74$, and 6 for the $75-84$ age groups. Women is a variable coded 1 for women and 0 for men.

Survey 2003 is coded 1 for the year 2003 and 0 for year 1995.

Region names are dummy variables coded 1 for the cited region and 0 for other geographical areas.

The latent trait was higher the West and South West regions $(+0.11)$ than in Paris Basin, and was lower in the North and Western Paris Basin regions (-0.17), and to a lesser extent in the East and Eastern Paris Basin regions $(-0.09)$. The age trend was more pronounced in the North, West and South West regions than in the Paris Basin relative to the 18-34 year-old group, -0.13 for the $35-44,-0.27$ for the $45-64,-0.41$ for the $65-74$, and -0.82 for the $75-84$ year-old groups.

Figure 2c reports the estimated values of the mean of the latent trait "Mental Health" as a function of the age categories, for men and women in 1995 and 2003, in the Paris Basin.

The variance in the reference group (men, aged 18-34, Paris, 1995) was estimated to be 1.68 and was lower in all the other regions with the exception of region 9 (Mediterranean Basin). The variance increased with the age $(+0.11$ when the recoded variable age increased of 1$)$.

\section{Discussion}

We describe here a methodology based on the latent regression Partial Credit Model, a model of the Rasch family adapted to polytomous items. Our model allows a latent trait to be explained by external covariates while detecting and handling items presenting DIF, and handling missing values. These last two features are essential to provide measure invariance, because 1) all measures of the latent concept are on the same scale, whatever the group in which the subject is classified, even if perception or functioning of one or several items differ between groups; and 2) the measures of the latent concept are independent of the set of observed items, and can be constructed even in presence of missing responses. As a practical example of the usefulness of this type of method, we applied it to determine whether the worsening trends and regional heterogeneity previously reported in France for HRQoL [3] could be confirmed after obtaining measure invariance.

One major finding of this study was the general decrease of HRQoL in France between 1995 and 2003, as assessed using three dimensions of HRQoL, and after adjustment for region, age and gender variables. The decrease was observed for both genders, all age categories and all the regions considered, without exception. For one dimension (General Health) a significant interaction was found which reinforced the decrease of the latent trait as a function of age category, but did not fully explain the decrease of the latent trait between the two years. Thus, after adjusting for age and gender, we observed a significant decrease of all three quality of life dimensions between 1995 and 2003. The decreases were of moderate size (standardized difference of 0.13 for $\mathrm{GH}$, 0.08 for $\mathrm{PF}$ and 0.14 for $\mathrm{MH}$ ), but it is remarkable that these decreases were systematic for both sexes, all age 


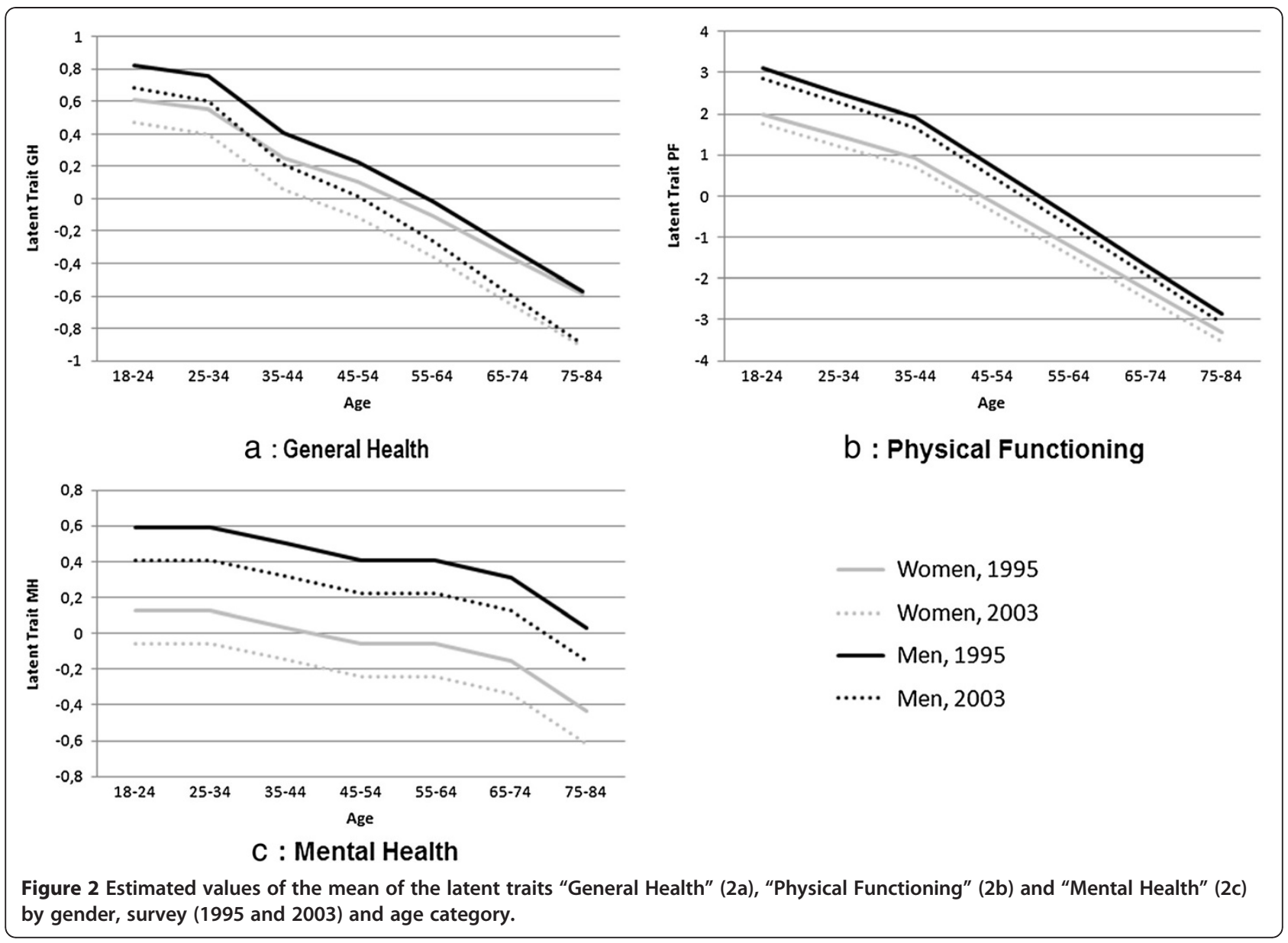

categories considered and all regions of France (see [3] for a full discussion of this results and limits to interpretation).

We tested for DIF for each item on main covariates. The creation of pseudo items for each level of the variables presenting DIF was used as a straightforward means of accounting for DIF. This is an improvement over the standard use of the scores of the SF36 which does not allow the DIF to be taken into account.

The DIF analysis conducted with the Partial Credit Model also revealed differences for gender or age variables that are easy to interpret: between the two genders, DIF was detected for the item "lift, carry groceries" (PF3); between the age categories, DIF was detected for items relating to general health perception (GH1, GH11a), physical activities (PF6) and work (MH1). Note that no DIF was detected between regions. This result is consistent with the homogeneity of French culture and language across France. By contrast, the DIF detected between the two samples (1995 and 2003) for items PF3 and PF45 is more difficult to interpret, but was, in both cases, only moderate.
Our findings are consistent with established processes concerning differences in quality of life dimensions between age categories and the sexes: HRQoL decreases with age, and is lower for women. Our analysis can be compared with the analysis of the scores as a function of the covariates, using linear regressions [3]. Trends associated with ageing, differences between genders and the decrease between 1995 and 2003 were found in this previous analysis. Concerning the differences between regions, the IRT approach seems to be more powerful, detecting both more and smaller differences between regions. Also, a larger part of variance was explained by the covariates in the IRT approach than in standard linear regression analyses using classical scores $(13 \%$ vs $11 \%$ for $\mathrm{GH}, 27 \%$ vs $20 \%$ for PF et $5 \%$ vs $3 \%$ for $\mathrm{MH}$ ); this may be due to the detection of a larger number of significant effects associated with regional disparities.

The use of a mixed model of the Rasch family also facilitated handling the problem of missing data in the questionnaire. Indeed, with the specific objectivity of the Rasch family models, the estimations of the parameters 
linked to the latent trait are consistent whatever the set of items that individuals responded to, whatever the nature (informative or not) of the missing data process [30-32]. In our study, the process of missing data was not at random [33] and therefore could not be ignored. Indeed, if we imputed all the missing data with Personal Mean Score (PMS) (without restriction on the rule of $50 \%$ of observed responses), we observe (imputed) mean scores on the General Health dimension of 68.01 for the 22981 individuals (87.1\%) without missing data, 65.95 for the 1842 individuals (7.0\%) with one or two missing items, and only 56.96 for the 1565 individuals (5.9\%) with three or more missing items. The difference between these three means is very significant $(\mathrm{p}<0.0001)$ and indicates that the missing data process is very informative. In a classical approach, the 1565 individuals with three or more missing items would not be analysed, and consequently, the latent variable measured by the score would have been overestimated. Also, important assumptions would be made for the 1842 individuals with one or two missing items, although the recommended imputation method (Personal Mean Score) is robust compared to more sophisticated methods such as multiple imputation [34,35].

Our analysis also clearly shows how latent regression models could be used to explain the latent variables by external (predictive) covariates. In a mixed Partial Credit Model, covariates explaining the mean of the latent trait can indeed be simply introduced into the model. This model is more powerful and gives results than are less biased than the method of using a traditional Partial Credit Model to estimate the individual values of the latent trait in a first step, and then in a second step to analyse these individual values with a linear model [36]. Last, in this mixed approach, the homoscedasticity assumption could be relaxed, by explaining the value of the variance of the latent trait by covariates.

Our work raises various issues about the Rasch model approach used. The Partial Credit Models required several assumptions, in particular the three fundamental assumptions of the Item Response Theory (IRT): local independence, monotonicity and unidimensionality. Mokken models gave a good fit to the data, such that we can be confident about the respect of these three assumptions. More, the fit of the Partial Credit Models were satisfactory when Differential Item Functioning (DIF) on some items was taken into account. Previous analyses of the SF36 using Rasch analyses recommend to be vigilant to the problem of local independence of the items of this questionnaire [13], but pointed out the interest of this approach to handle missing data [14].

Three mains limits of this analysis can be noted. First, no data about the health (physical or mental diseases, disabilities...) or the socio-economic context of the individuals (marital status, outcomes, employment...) were available. Consequently, the part of variance explained by the available covariates is limited: only $13 \%$ for $\mathrm{GH}$, $27 \%$ for PF and 5\% for $\mathrm{MH}$. Secondly, the fit of the model have been evaluated only for the model without covariates, because no adapted fit test exists. Last, the two samples (1995 and 2003) are not matched: there are not the same individuals that respond to two dates.

As an extension of this study, a hierarchical model could be envisaged, by considering macro socioeconomical variables relevant to the regions; this may improve the part of explained variance of the latent trait. Another potentially informative extension of this work would be the analysis of a new sample, to evaluate the evolution of Health-Related Quality of life in France since 2003.

\section{Additional files}

Additional file 1: Items of the three studied dimensions of the SF36.

Additional file 2: Recoding the Physical Functioning dimension.

\section{Competing interests}

The authors declare that they have no competing interests.

\section{Authors' contributions}

All authors have made substantial contributions to conception and design, analysis and interpretation of data. All authors read and approved the final manuscript.

\section{Author details}

'UPRES EA 4275 (SPHERE) « Biostatistics, Pharmacoepidemiology and Subjective Measures in Health Sciences », University of Nantes, Nantes, France. ${ }^{2}$ Plateforme de Biométrie, Direction de la Recherche Clinique, CHU de Nantes, Nantes, France. ${ }^{3}$ Nancy-Université, Université Paris-Descartes, Université Metz Paul Verlaine, Research unit APEMAC, EA 4360 Paris, France. ${ }^{4}$ Biostatistics and Epidemiology Unit, Assistance Publique-Hôpitaux de Paris, Hôtel Dieu, Paris, France. ${ }^{5}$ Department of History and Philosophy of Sciences, University of Paris Diderot - Paris 7, Paris, France.

Received: 5 April 2012 Accepted: 3 November 2012

Published: 28 November 2012

\section{References}

1. Zack MM, Moriarty DG, Stroup DF, Ford ES, Mokdad AH: Worsening trends in adult health-related quality of life and self-rated health-United States, 1993-2001. Public Health Rep. 2004, 119(5):493-505.

2. The South Australian Monitoring and Surveillance System (SAMSS) 2002: PROS | Population Research and Outcome Studies (PROS) [Internet]; 2005. Available de: http://health.adelaide.edu.au/pros/.

3. Audureau E, Rican S, Coste J: Worsening trends and increasing disparities in health-related quality of life: evidence from two French population-based cross sectional surveys, 1995-2003. Qual Life Res:; 2012. Published online.

4. Bacci S, Data Analysis and Classification: Latent Regression in Rasch Framework. Springer Berlin Heidelberg: Palumbo F, Lauro CN, Greenacre MJ, éditeurs; 2010:319-27.

5. Paek I, Wilson M: Formulating the Rasch Differential Item Functioning Model Under the Marginal Maximum Likelihood Estimation Context and Its Comparison With Mantel-Haenszel Procedure in Short Test and Small Sample Conditions. Educational and Psychological Measurement. 2011, 71(6):1023-1046. 
6. De BP, Wilson M: Explanatory Item Response Models. Springer: A Generalized Linear and Nonlinear Approach. 1er éd; 2004.

7. Adams R, Wilson M, Wu M: Multilevel item response modelling: An approach to errors in variables regression. Journal of Educational and Behavioral Statistics. 1997, 22:47-76

8. Daponte-Codina A, Bolívar-Muñoz J, Toro-Cárdenas S, Ocaña-Riola R, Benach-Rovira J, Navarro-López V: Area deprivation and trends in inequalities in self-rated health in Spain, 1987-2001. Scand J Public Health. 2008, 36(5):504-15.

9. Zahran HS, Kobau R, Moriarty DG, Zack MM, Holt J, Donehoo R: Health-related quality of life surveillance--United States, 1993-2002. MMWR Surveill Summ. 2005, 54(4):1-35.

10. Ware JE Jr, Sherbourne CD: The MOS 36-item short-form health survey (SF-36). I. Conceptual framework and item selection. Med Care 1992, 30(6):473-83.

11. Leplège $A$, Ecosse E, Pouchot J, Coste J, Perneger T: Le questionnaire MOS SF-36 - manuel de l'utilisateur et guide d'interprétation des scores. Paris: ERSTEM; 2001

12. Ware JE Jr, Gandek B: Overview of the SF-36 Health Survey and the International Quality of Life Assessment (IQOLA) Project. J Clin Epidemiol. 1998, 51(11):903-12

13. Horton M, Tennant A: Applying Rasch analysis to the SF-36 physical function scale: effect of dependent items. Trials. 2011, 12(Suppl 1):A75

14. Raczek AE, Ware JE, Bjorner JB, Gandek B, Haley SM, Aaronson NK, et al: Comparison of Rasch and summated rating scales constructed from SF-36 physical functioning items in seven countries: results from the IQOLA Project. International Quality of Life Assessment. J Clin Epidemiol 1998, 51(11):1203-14.

15. Masters G: A Rasch model for partial credit scoring. Psychometrika. 1982, 47(2):149-74.

16. Andrich D, Luo G: Conditional pairwise estimation in the Rasch model for ordered response categories using principal components. J Appl Meas. 2003, 4(3):205-21.

17. Sijtsma K, Molenaar IW: Introduction to Nonparametric Item Response Theory: Sage Publications, Inc; 2002.

18. Molenaar IW, Sijtsma K, Boer P: MSP5 for Windows: a program for Mokken scale analysis for polytomous items. iec ProGAMMA: version 5.0: user's manual; 2000.

19. Hambleton RK: Good practices for identifying differential item functioning. Med Care. 2006, 44(11 Suppl 3):S182-188.

20. Smith AB, Rush R, Fallowfield LJ, Velikova G, Sharpe M: Rasch fit statistics and sample size considerations for polytomous data. BMC Medical Research Methodology. 2008, 8(1):33.

21. Andrich D, Sheridan BS, Luo G: Rumm 2030: Rasch Unidimensional Measurement Models [computer software]. Perth, Western Australia: RUMM Laboratory; 2010.

22. Linacre J: Sample Size and Item Calibration Stability. Rasch Measurement Transactions. 1994, 7(4):328.

23. Reise $\mathrm{S}, \mathrm{Yu} \mathrm{J}$ : Parameter recovery in the graded response model using MULTILOG. Journal of Educational Measurement. 1990, 27(2):133-44.

24. Akaike $\mathrm{H}$ : A new look at the statistical model identification. IEEE Transactions on Automatic Control. 1974, 19(6):716-723.

25. Muller S, Thomas E, Peat G: Derivation and testing of an interval-level score for measuring locomotor disability in epidemiological studies of middle and old age. Qual Life Res. 2009, 18(10):1341-55.

26. StataCorp LP: Stata Statistical Software: Release 11. College Station, TX: StataCorp LP; 2009.

27. Hardouin J-B, Bonnaud-Antignac A, Sébille V: Nonparametric item response theory using Stata. Stata Journal. 2011, 11(1):30-51.

28. SAS Institute Inc: Procedures Guide. Cary, NC: SAS Institute Inc; 2008

29. Hardouin J-B, Mesbah M: The SAS Macro-Program \%AnaQol to Estimate the Parameters of Item Responses Theory Models. Communications in Statistics - Simulation and Computation. 2007, 36:437-53.

30. Blanchin M, Hardouin JB, Le Néel T, Kubis G, Sébille V: Analysis of longitudinal Patient-Reported Outcomes with informative and non-informative dropout: Comparison of CTT and Rasch-based methods. International Journal of Applied Mathematics \& Statistics [Internet] 2011, 24:I-11. Available de: http://www.ceserp.com/cp-jour/index php?journal=ijamas\&page=article\&op=view\&path[ $=1004$.

31. Hardouin J-B, Conroy R, Sébille V: Imputation by the mean score should be avoided when validating a Patient Reported Outcomes questionnaire by a Rasch model in presence of informative missing data. BMC Med Res Methodol. 2011, 11:105.

32. Hamel J-F, Hardouin J-B, Le Néel T, Kubis G: Sébille V. Submited: Rasch models to detect a group effect for Patients Reported Outcomes with possibly informative missing data; 2012.

33. Peyre $\mathrm{H}$, Coste $\mathrm{J}$, Leplège A: Identifying type and determinants of missing items in quality of life questionnaires: Application to the SF-36 French version of the 2003 Decennial Health Survey. Health Qual Life Outcomes. 2010, 8:16.

34. Peyre $H$, Leplège $A$, Coste J: Missing data methods for dealing with missing items in quality of life questionnaires. A comparison by simulation of personal mean score, full information maximum likelihood, multiple imputation, and hot deck techniques applied to the SF-36 in the French 2003 decennial health survey. Quality of Life Research. 2010 20(2):287-300

35. Hamel J-F, Hardouin J-B, Le Néel T, Kubis G, Roquelaure Y, Sébille V: Study of different methods for comparing groups by analysis of subjective health measurements. PLOS ONE 2012, 7(10):e44695.

36. Blanchin M, Hardouin J-B, Le Neel T, Kubis G, Blanchard C, Mirallié E, et al: Comparison of CTT and Rasch-based approaches for the analysis of longitudinal Patient Reported Outcomes. Stat Med 2011, 30(8):825-38.

\section{doi:10.1186/1471-2288-12-182}

Cite this article as: Hardouin et al: Spatio-temporal Rasch analysis of quality of life outcomes in the French general population. Measurement invariance and group comparisons. BMC Medical Research Methodology 2012 12:182.

\section{Submit your next manuscript to BioMed Central and take full advantage of:}

- Convenient online submission

- Thorough peer review

- No space constraints or color figure charges

- Immediate publication on acceptance

- Inclusion in PubMed, CAS, Scopus and Google Scholar

- Research which is freely available for redistribution 